\title{
Purely imaginary eigenvalues of Zakharov-Shabat systems
}

\author{
M. Klaus and J. K. Shaw \\ Department of Mathematics, Virginia Tech, Blacksburg, Virginia 24061-0123
}

(Received 9 August 2001; published 11 February 2002)

\begin{abstract}
Zakharov-Shabat systems with single-hump and real, but not necessarily symmetric, potentials are shown to have purely imaginary eigenvalues only. Coupled with examples of double-hump potentials with nonimaginary eigenvalues, this establishes that confinement of Zakharov-Shabat eigenvalues to the imaginary axis is a characteristic of potentials whose energy is concentrated in a single region of the time axis.
\end{abstract}

DOI: 10.1103/PhysRevE.65.036607

PACS number(s): 42.65.Tg, 42.65.Wi

\section{INTRODUCTION}

Zakharov-Shabat (ZS) systems are sets of coupled scalar first-order differential equations of the form $[1,2]$

$$
\nu_{1}^{\prime}=-i \xi \nu_{1}+q(t) \nu_{2}, \quad \nu_{2}^{\prime}=i \xi \nu_{2}-q(t)^{*} \nu_{1}
$$

where $\xi$ is a complex eigenvalue parameter and the asterisk denotes the complex conjugate. In Eq. (1) the potential $q(t)$ is, in general, a complex valued function of the real variable $t$. An eigenvalue (EV) of Eq. (1) is a complex number $\xi$, with $\operatorname{Im}(\xi)>0$, for which Eq. (1) has a nontrivial solution vector

$$
\vec{\nu}(t)=\left(\begin{array}{l}
\nu_{1}(t) \\
\nu_{2}(t)
\end{array}\right)
$$

of integrable square on the real line, i.e., $\int_{-\infty}^{\infty}\left[\left|\nu_{1}(t)\right|^{2}\right.$ $\left.+\left|\nu_{2}(t)\right|^{2}\right] d t<\infty$.

To motivate ZS systems, they have their origin in the inverse scattering transform (IST) theory associated with the nonlinear Schrödinger equation (NLSE), which we may write in the form $[1,2]$

$$
i u_{z}+(1 / 2) u_{t t}+|u|^{2} u=0, \quad u=u(z, t) .
$$

In nonlinear fiber optics, $u(z, t)$ in Eq. (2) represents the slowly varying field of a pulse propagating in a lossless optical fiber under conditions of anomalous group velocity dispersion and non-negligible fiber nonlinearities [3]. Here $z$ is a normalized propagation length and $t$ is local pulse time. The connection with Eq. (1) is that the NLSE (2) can theoretically be solved, although in intractable form, by setting $q(t)=u(0, t)$ in Eq. (1) and solving for $u(z, t)$ by the IST procedure associated with Eq. (1) [1,2]. As for the variable $z$, it is a central result in the method that the EVs and other spectral quantities evolve in a specific and known way as functions of $z[1,2]$. In particular, the EVs are independent of $z$. This leads to a complicated analytical formula for $u(z, t)$ in terms of the EVs $\xi_{k}(k=1,2,3, \ldots)$ of Eq. (1) and additional scattering quantities including the reflection coefficients and norming constants [1,2]. ZS systems (1) are often written with a potential $u(z, t)$ instead of $q(t)$. Since we are interested only in EVs, which are independent of $z$, we suppress the $z$ dependence.

We note that Eq. (1) is a non-self-adjoint EV problem and that Eq. (2) is known as the focusing version of the NLSE. The corresponding self-adjoint problem has a plus sign be- fore the term $q(t)^{*}$ in Eq. (1), a minus sign before the $(1 / 2) u_{t t}$ term in Eq. (2), and is called the nonfocusing or defocusing case.

For physically realistic pulse profiles $q(t)$ there are a finite number of EVs $\xi_{k}$, which correspond to optical soliton solutions of Eq. (2). The real and imaginary parts of the $\xi_{k}$ are linked with relative soliton velocities and amplitudes, respectively [2]. Generically, but not always, the EVs lie on the positive imaginary axis; i.e., $\xi_{k}=i \sigma_{k}, \sigma_{k}>0$. This property is physically significant because a pair of solutions with identical real parts travel with the same velocity and can combine to form a higher-order periodic soliton (periodic in the $z$ variable) [2]. For the standard real valued symmetric pulse shapes $q(t)$, including hyperbolic secants, Gaussians, and rectangles, all the EVs are on the positive imaginary axis [4-12].

In fact, an interesting "folklore" property seems to have arisen in the literature over the last 25 years, namely, that only purely imaginary EVs can occur for symmetric real valued potentials $q(t)$. This property has been claimed explicitly several times $[6,7,13]$ (and in Ref. [14] for merely real potentials). However, in a recent paper [12] the present authors provided examples of symmetric, real valued potentials with nonimaginary EVs. The examples in Ref. [12] were symmetric piecewise constant functions consisting of two or more "humps," or lobes, or regions of focus of pulse energy (area under the curve of $|q(t)|^{2}$ ). The standard potentials (hyperbolic secant, Gaussian, etc.), together with the doublelobe examples, suggest that the confinement of EVs to the imaginary axis may be connected with the extent to which pulse energy is concentrated in some region of the $t$ axis. In the next section we derive precisely this result: single-lobe real potentials have purely imaginary EVs only. Symmetry of $q(t)$ is not a factor. For completeness we cite another example of a two-lobe potential with EVs off the imaginary axis; this example is piecewise quadratic. The single-lobe theorem and the double-lobe examples, which are the main contributions of this paper, close a loop in the somewhat ambiguous history of this subject.

We derive the single-lobe result in Sec. III, following some preliminaries in Sec. II. We will assume that $q(t)$ in Eq. (1) is non-negative, bounded, piecewise smooth and integrable on the real line $\left[q \in L^{1}(-\infty, \infty)\right]$, but the key condition is simply that $q(t)$ be nondecreasing on the left of $t$ $=0$ and nonincreasing on the right (thus motivating the term "single lobe" potential). Since Eq. (1) is invariant under 
shifts of the $t$ variable, no generality is lost in taking the point of concentration of pulse energy to be $t=0$. We will suppose $q(0)>0$, as otherwise $q(t)$ would vanish identically. By piecewise smooth we mean that $q(t)$ and $q^{\prime}(t)$ have finite left and right limits for all $t$ and that in any bounded interval $q(t)$ has at most finitely many jump discontinuities.

After giving the proof in Sec. III, we discuss in Sec. IV the closely connected phenomenon of double EVs on the imaginary axis. We close the paper in Sec. V with some remarks and a summary. There is an Appendix containing a technical derivation.

\section{PRELIMINARIES}

In scattering theory one defines the Jost solutions $\vec{\psi}(t, \xi)$ and $\vec{\varphi}(t, \xi)$ of Eq. (1) by the asymptotic properties [1]

$$
\begin{gathered}
\vec{\psi}(t, \xi) \cong\left(\begin{array}{l}
0 \\
1
\end{array}\right) e^{i \xi t}, \quad t \rightarrow \infty, \quad \vec{\varphi}(t, \xi) \cong\left(\begin{array}{l}
1 \\
0
\end{array}\right) e^{-i \xi t}, \\
t \rightarrow-\infty, \quad \operatorname{Im}(\xi)>0
\end{gathered}
$$

where

$$
\vec{\psi}=\left(\begin{array}{c}
\psi_{1} \\
\psi_{2}
\end{array}\right), \quad \vec{\varphi}=\left(\begin{array}{c}
\varphi_{1} \\
\varphi_{2}
\end{array}\right)
$$

Up to constant multiples, $\vec{\psi}(t, \xi)$ and $\vec{\varphi}(t, \xi)$ are the unique solutions that are integrable on $[0, \infty)$ and $(-\infty, 0]$, respectively. An EV of Eq. (1) thus corresponds to a solution

$$
\vec{\nu}(t)=\left(\begin{array}{l}
\nu_{1}(t) \\
\nu_{2}(t)
\end{array}\right)
$$

that is a constant multiple of each of $\vec{\psi}(t, \xi)$ and $\vec{\varphi}(t, \xi)$. Using the integral equation versions of Eq. (1), one can show that $\vec{\psi}(t, \xi)$ and $\vec{\varphi}(t, \xi)$ are the unique solutions to the coupled pairs of integral equations

$$
\begin{gathered}
\psi_{1}(t)=-e^{-i \xi t} \int_{t}^{\infty} q(\tau) e^{i \xi \tau} \psi_{2}(\tau) d \tau, \\
\psi_{2}(t)=e^{i \xi t}\left[1+\int_{t}^{\infty} q(\tau) e^{-i \xi \tau} \psi_{1}(\tau) d \tau\right], \\
\varphi_{1}(t)=e^{-i \xi t}\left[1+\int_{-\infty}^{t} q(t) e^{i \xi \tau} \varphi_{2}(\tau) d \tau\right], \\
\varphi_{2}(t)=-e^{i \xi t} \int_{-\infty}^{t} q(\tau) e^{-i \xi \tau} \varphi_{1}(\tau) d \tau,
\end{gathered}
$$

where we have suppressed the dependence on $\xi$. Since the eigenfunctions (EFs) $\vec{\nu}(t)$ are multiples of $\vec{\psi}(t, \xi)$ and $\vec{\varphi}(t, \xi)$ then each $\vec{\nu}(t)$ satisfies Eq. (3), with the 1's being replaced by possibly different constants.
We require some bounds on the EFs. Let $\xi=\alpha+i \beta, \beta$ $>0$ be an EV. By standard arguments involving the Gronwall inequality and Eq. (3), one can show that $\nu_{2}$ satisfies a bound of type

$$
\left|\nu_{2}(t)\right| \leqslant C e^{-\beta t}
$$

for some constant $C>0$. Inserting Eq. (4) into the first of Eq. (3) (for $\vec{v}$ ) shows that $\nu_{1}$ satisfies

$$
\left|\nu_{1}(t)\right| \leqslant C e^{\beta t} \int_{t}^{\infty} q(\tau) e^{-2 \beta \tau} d \tau
$$

Estimates (4) and (5) will be useful only for $t \rightarrow \infty$ but it is straightforward to obtain analogous bounds of the form

$$
\left|\nu_{1}(t)\right| \leqslant C e^{\beta t}, \quad\left|\nu_{2}(t)\right| \leqslant C e^{-\beta t} \int_{-\infty}^{t} q(\tau) e^{2 \beta \tau} d \tau
$$

which are pertinent for $t \rightarrow-\infty$, where we can use the same constant $C$ for Eqs. (4)-(6). In particular, $\vec{\nu}(t) \rightarrow \overrightarrow{0},|t| \rightarrow \infty$. But more is true for single-lobe potentials. Let $t>0$ and suppose $q(t)>0$. By Eq. (5)

$$
\begin{aligned}
\frac{\left|\nu_{1}(t)\right|^{2}}{q(t-)} & \leqslant \frac{\left|\nu_{1}(t)\right|^{2}}{q(t)} \leqslant \frac{C^{2} e^{2 \beta t}\left(\int_{t}^{\infty} q(\tau) e^{-2 \beta \tau} d \tau\right)^{2}}{q(t)} \\
& \leqslant C^{2} e^{2 \beta t}\left(\int_{t}^{\infty} e^{-2 \beta \tau} d \tau\right)\left(\int_{t}^{\infty} q(\tau) e^{-2 \beta \tau} d \tau\right) \\
& =\left(C^{2} / 2 \beta\right)\left(\int_{t}^{\infty} q(\tau) e^{-2 \beta \tau} d \tau\right) \\
& \leqslant \frac{C^{2} q(t+) e^{-2 \beta t}}{4 \beta^{2}}
\end{aligned}
$$

[where $q(t \pm)$ denotes a right-left-hand-side limit] since $q(t)$ is nonincreasing. Thus, $\left|\nu_{1}(t)\right| \leqslant(C / 2 \beta) q(t) e^{-\beta t}$. Using Eq. (6) one can show that completely analogous results hold for the quotient $\left|\nu_{2}(t)\right|^{2} / q(t)$ with $t<0, q(t)>0$.

We will also need an alternate condition implying purely imaginary EVs. Write Eq. (1) in the form

$$
i \nu_{1}^{\prime}-i q(t) \nu_{2}=\xi \nu_{1}, \quad i \nu_{2}^{\prime}+i q(t) \nu_{1}=-\xi \nu_{2} .
$$

Assuming that $\xi$ is an EV, multiply the first of Eq. (8) by $\nu_{2}^{*}$, the second by $\nu_{1}^{*}$ and subtract to obtain

$$
i\left(\nu_{1}^{\prime} \nu_{2}^{*}-\nu_{2}^{\prime} \nu_{1}^{*}\right)-i q(t)\left(\left|\nu_{1}\right|^{2}+\left|\nu_{2}\right|^{2}\right)=\xi\left(\nu_{1} \nu_{2}^{*}+\nu_{2} \nu_{1}^{*}\right)
$$

Integrating Eq. (9) over the real $t$ axis we have

$$
\begin{aligned}
& i \int_{-\infty}^{\infty}\left(\nu_{1}^{\prime} \nu_{2}^{*}-\nu_{2}^{\prime} \nu_{1}^{*}\right) d t-i \int_{-\infty}^{\infty} q(t)\left(\left|\nu_{1}\right|^{2}+\left|\nu_{2}\right|^{2}\right) d t \\
& \quad=\xi \int_{-\infty}^{\infty}\left(\nu_{1} \nu_{2}^{*}+\nu_{2} \nu_{1}^{*}\right) d t
\end{aligned}
$$

Integrating the first term in Eq. (10) by parts and using the fact that $\left|\nu_{1}\right|,\left|\nu_{2}\right| \rightarrow 0,|t| \rightarrow \infty$, one sees that the first integral in Eq. (10) equals $\left(\int_{-\infty}^{\infty}\left(\nu_{1}^{\prime} \nu_{2}^{*}-\nu_{2}^{\prime} \nu_{1}^{*}\right) d t\right)^{*}$, i.e., its own 
conjugate. Thus the first integral in Eq. (10) is real, so the left-hand side of Eq. (10) is purely imaginary. The integral on the right-hand side of Eq. (10) is also real. Therefore, if

$$
\int_{-\infty}^{\infty}\left(\nu_{1} \nu_{2}^{*}+\nu_{2} \nu_{1}^{*}\right) d t \neq 0
$$

then $\xi$ must be purely imaginary. We will use Eq. (11) in deriving the single-lobe result.

In Ref. [7] the authors note that the condition (11) implies purely imaginary EVs but only indicate that the integral typically does not vanish unless $q(t)$ is antisymmetric.

\section{SINGLE-LOBE POTENTIALS}

Still assuming that $\xi=\alpha+i \beta, \beta>0$, is an EV of Eq. (1) we will show that $\xi$ must be purely imaginary. Multiply the first of Eq. (1) by $\nu_{1}^{*}$ to obtain $\nu_{2} \nu_{1}^{*}=[1 / q(t)] \nu_{1}^{\prime} \nu_{1}^{*}$ $+[i \xi / q(t)]\left|\nu_{1}\right|^{2}$, assuming $q(t)>0$. The conjugate of this expression is $\nu_{1} \nu_{2}^{*}=[1 / q(t)] \nu_{1}^{\prime *} \nu_{1}-\left[i \xi^{*} / q(t)\right]\left|\nu_{1}\right|^{2}$. Adding and integrating over an interval $[0, d]$ where $q(t)>0$, we have

$$
\begin{aligned}
\int_{0}^{d}\left(\nu_{2} \nu_{1}^{*}+\nu_{1} \nu_{2}^{*}\right) d t= & \int_{0}^{d}[1 / q(t)]\left(\nu_{1}^{\prime} \nu_{1}^{*}+\nu_{1}^{\prime *} \nu_{1}\right) d t \\
& +i\left(\xi-\xi^{*}\right) \int_{0}^{d} \frac{\left|\nu_{1}\right|^{2}}{q(t)} d t
\end{aligned}
$$

Using Eq. (12), and a corresponding integral over $(c, 0), c$ $<0$, we are going to show that Eq. (11) holds, with the integral being strictly negative. Working first with Eq. (12), it will be convenient to break the argument into cases depending on whether $q(t)$ does or does not have compact support on $[0, \infty)$. Suppose $q(t)$ has compact support. Then there is a unique positive $d_{0}$ such that $q\left(d_{0}^{+}\right)=0$ and $q(t)>0$ for 0 $<t<d_{0}$. Taking $d=d_{0}$ in Eq. (12), Eq. (7) shows that the integrals on the right-hand side of Eq. (12) exist even if $q\left(d_{0}^{-}\right)=0$. The first integral on the right-hand side of Eq. (12), which equals $\int_{0}^{d}\left[\left(\nu_{1} \nu_{1}^{*}\right)^{\prime} / q(t)\right] d t$, can be integrated by parts as

$$
\left.\sum_{k=1}^{N+1} \frac{\left|\nu_{1}(s)\right|^{2}}{q(s)}\right|_{\left(t_{k-1}\right)^{+}} ^{\left(t_{k}\right)^{-}}+\int_{0}^{d} \frac{\left|\nu_{1}\right|^{2} q^{\prime}(t)}{q(t)^{2}} d t,
$$

where $t_{0}=0, t_{k}(1 \leqslant k \leqslant N)$ are the jump discontinuities of $q(t)$ in $[0, d)$ and $t_{N+1}=d \geqslant t_{N}$, and where the integral in Eq. (13) also exists by Eq. (7). The upper $k=N+1$ term in Eq. (13) is the left-hand-side limit

$$
\lim _{l \rightarrow a^{-}}\left[\left|\nu_{1}(t)\right|^{2} / q(t)\right]
$$

which vanishes by the last integral in Eq. (7) because $q\left(d_{0}^{+}\right)=0$. Rearranging the sum in Eq. (13), and substituting Eq. (13) into Eq. (12) gives

$$
\begin{aligned}
\int_{0}^{d}\left(\nu_{2} \nu_{1}^{*}+\nu_{1} \nu_{2}^{*}\right) d t= & -\frac{\left|\nu_{1}(0)\right|^{2}}{q(0)}+\sum_{k=1}^{N}\left(\frac{\left|\nu_{1}\left(t_{k}^{-}\right)\right|^{2}}{q\left(t_{k}^{-}\right)}\right. \\
& \left.-\frac{\left|\nu_{1}\left(t_{k}^{+}\right)\right|^{2}}{q\left(t_{k}^{+}\right)}\right)+\int_{0}^{d} \frac{\left|\nu_{1}\right|^{2} q^{\prime}(t)}{q(t)^{2}} d t \\
& -2 \beta \int_{0}^{d} \frac{\left|\nu_{1}\right|^{2}}{q(t)} d t
\end{aligned}
$$

where each term in the summation in Eq. (14) is nonpositive because $\nu_{1}(t)$ is continuous and $q\left(t_{k}^{+}\right) \leqslant q\left(t_{k}^{-}\right)$. Indeed, the right-hand side of Eq. (14) is negative since $q^{\prime}(t) \leqslant 0$ and $\beta>0$. Since $\nu_{1}(t)=0$ for $t>d=d_{0}$ by Eq. (7), then the lefthand side of Eq. (14) equals the same expression with $d$ $=\infty$. This shows that $\int_{0}^{\infty}\left(\nu_{1} \nu_{2}^{*}+\nu_{2} \nu_{1}^{*}\right) d t<0$ in the compact support case on $[0, \infty)$. On the other hand, if $q(t)>0$ for all $t$ (noncompact support) then the right-hand side of Eq. (14) is the same except that the term $\left|\nu_{1}(d)\right|^{2} / q\left(d^{-}\right)$must now be included. The limit of this term as $d \rightarrow \infty$ is 0 by Eq. (7) and so letting $d \rightarrow \infty$ in Eq. (14) gives $\int_{0}^{\infty}\left(\nu_{1} \nu_{2}^{*}+\nu_{2} \nu_{1}^{*}\right) d t<0$ in this case as well.

Turning to the interval $[c, 0]$ we multiply the second of Eq. (1) by $\nu_{2}^{*}$ and proceed as above to obtain this time

$$
\begin{aligned}
\int_{c}^{0}\left(\nu_{2} \nu_{1}^{*}+\nu_{1} \nu_{2}^{*}\right) d t= & -\left.\frac{\left|\nu_{2}\right|^{2}}{q(t)}\right|_{c} ^{0}-\int_{c}^{0} \frac{\left|\nu_{2}\right|^{2} q^{\prime}(t)}{q(t)^{2}} d t \\
& -2 \beta \int_{c}^{0} \frac{\left|\nu_{2}\right|^{2}}{q(t)} d t
\end{aligned}
$$

where the integrated expression $\left.(*)\right|_{c} ^{0}$ includes jump terms that we do not write out explicitly here. Reasoning as before, $\left|\nu_{2}(c)\right|^{2} / q(c+) \rightarrow 0, c \rightarrow-\infty$, and $q^{\prime}(t) \geqslant 0$ in Eq. (15), making the right-hand side of Eq. (15) strictly negative. Summing the two halves establishes Eq. (11), where the integral is strictly negative. This proves the following.

Single-lobe theorem. Let $q(t)$ be a non-negative, piecewise smooth, bounded $L^{1}$ function on the real line such that $q(t)$ is nondecreasing for $t<0$ and nonincreasing for $t>0$. Then any EVs of Eq. (1) must be purely imaginary.

Examples. For the specific function $q_{1}(t)=\kappa[-1$ $\left.+(3 \pi / 4)+3 t^{2}\right],-1 \leqslant t \leqslant 1, q_{1}(t)=0$ otherwise, one may compute numerically that there is a pair of nonimaginary EVs located symmetrically about the imaginary axis in the upper-half plane when $\kappa$ is in the range $0.956<\kappa<0.9999$. As $\kappa$ increases the EVs approach each other and the imaginary axis, and coalesce into a double $\mathrm{EV}$ at $\xi=0.03 i$ when $\kappa=0.9999$. The graph of $q_{1}(t)$ is a cutoff quadratic, which one can think of as a two-lobe potential. If the parameter $\kappa$ is reversed, then we have a pair of nonimaginary EVs born as an imaginary pair collide and transition through a double EV state. The example $q_{2}(t)=145.85$, for $1.06 \leqslant|t| \leqslant 1.07$, $q_{2}(t)=0$ otherwise, which has an $\mathrm{EV}$ at $1.4426+i$, was cited in Ref. [12]. 


\section{EXTENSIONS}

We turn first to the occurrence of double EVs on the imaginary axis, a phenomenon that is closely linked with the existence of nonimaginary EVs. Recall the example of the piecewise quadratic two-lobe potential mentioned at the end of Sec. III where a pair of nonimaginary EVs move towards each other in the upper-half plane and collide, as a parameter $\kappa$ is increased, on the imaginary axis to form a double EV. This suggests that single-lobe potentials have no double EVs on the imaginary axis, which we now establish. To simplify the argument we will suppose that $q(t)$ is continuous and vanishes outside $[-d, d]$.

The Wronskian of two solutions $\vec{\nu}^{(1)}(t, \xi)$ and $\vec{\nu}^{(2)}(t, \xi)$ of Eq. (1) is defined as

$$
W\left[\vec{\nu}^{(1)}, \vec{\nu}^{(2)}\right](\xi)=\nu_{1}^{(1)}(t, \xi) \nu_{2}^{(2)}(t, \xi)-\nu_{2}^{(1)}(t, \xi) \nu_{1}^{(2)}(t, \xi) .
$$

It is simple to verify that the Wronskian of two solutions is constant and vanishes if and only if the solutions are linearly dependent. Then EVs are complex numbers $\xi$ where $W[\vec{\psi}, \vec{\varphi}](\xi)=0$. A double $\mathrm{EV}$ is defined as an $\mathrm{EV} \xi$ where additionally $(d / d \xi) W[\vec{\psi}, \vec{\varphi}](\xi)=0$, so as to form a double zero of the Wronskian. In the Appendix we show that $\xi$ is a double EV if and only if

$$
\int_{-\infty}^{\infty} \nu_{1}(t, \xi) \nu_{2}(t, \xi) d t=0
$$

where $\vec{\nu}(t, \xi)$ is the corresponding eigenfunction.

As in the purely imaginary EV case in connection with Eq. (7), we can thus rule out double imaginary EVs by showing that the integral in Eq. (16) does not vanish. In fact, let $\xi=i \beta, \beta>0$, be an EV and suppose that $q(t)$ satisfies the hypotheses of the single-lobe theorem (but is continuous and supported on $[-d, d]$ ). System (1) for the eigenfunction $\vec{\nu}(t, \xi)$ becomes

$$
\nu_{1}^{\prime}=q(t) \nu_{2}+\beta \nu_{1}, \quad \nu_{2}^{\prime}=-q(t) \nu_{1}-\beta \nu_{2} .
$$

Multiply the first of Eq. (17) by $\nu_{1}$, solve for $\nu_{1} \nu_{2}$, and integrate over $[0, d]$ to obtain

$$
\begin{aligned}
\int_{0}^{d} \nu_{1}(t) \nu_{2}(t) d t= & -\frac{\nu_{1}(0)^{2}}{2 q(0)}+\frac{1}{2} \int_{0}^{d} \frac{\nu_{1}^{2} q^{\prime}(t)}{q(t)^{2}} d t \\
& -\beta \int_{0}^{d} \frac{\nu_{1}^{2}}{q(t)} d t
\end{aligned}
$$

where the argument is the same as that leading to Eq. (14). The right-hand side of Eq. (18) is negative by the assumptions on $q(t)$. Using the second of Eq. (17) and integrating over $[-d, 0]$ yields an analogous expression for the integral over $[-d, 0]$. Adding gives $\int_{-\infty}^{\infty} \nu_{1}(t, \xi) \nu_{2}(t, \xi) d t<0$ that rules out double imaginary EVs by Eq. (16). The extensions to the noncompact support and piecewise smooth cases are simple and proceed as above.

The relationship of the one-lobe theorem to the two-lobe examples makes it seem, on one hand, that the theorem is not capable of much improvement. On the other hand, we note that Eqs. (14) and (15) together yield (assume that $q(t)$ is continuous and has compact support $[-d, d]$ for simplicity)

$$
\begin{aligned}
\int_{-d}^{d}\left(\nu_{2} \nu_{1}^{*}+\nu_{1} \nu_{2}^{*}\right) d t= & -\frac{\left|\nu_{1}(0)\right|^{2}+\left|\nu_{2}(0)\right|^{2}}{q(0)} \\
& -\int_{0}^{d} \frac{\left|\nu_{1}\right|^{2}}{q(t)}\left(2 \beta-\frac{q^{\prime} t}{q(t)}\right) d t \\
& -\int_{-d}^{0} \frac{\left|\nu_{2}\right|^{2}}{q(t)}\left(2 \beta+\frac{q^{\prime}(t)}{q(t)}\right) d t .
\end{aligned}
$$

Any condition on $q(t)$ making this expression negative for all $\beta>0$ forces purely imaginary EVs by Eq. (11). In another direction, note that a condition of the form $\left|q^{\prime}(t) / q(t)\right| \leqslant K$ implies that the above equation is negative for $2 \beta>K$; that is, there are no nonimaginary EVs $\xi$ with $\operatorname{Im}(\xi)>(K / 2)$.

The proof given in this paper for the single-lobe theorem can be replaced by more elegant perturbation theory arguments, based on perturbations of the compact support case.

\section{SUMMARY AND CONCLUDING REMARKS}

By deriving the single-lobe result, and citing the doublelobe examples, we have established that purely imaginary EVs are a feature of potentials with concentrated energy, symmetry of the potential not being a factor.

The notation in Eq. (1) reflects the possibility that $q(t)$ can be complex valued, although we have taken $q(t)$ to be real (and non-negative) in this paper. However, the case where $q(t)$ is complex, and especially of the form $q(t)$ $=q_{0}(t) e^{i p(t)}, q_{0}(t)>0$ and $p(t)$ real, is the physically important situation of "chirped" optical pulses [3]. In Ref. [12] these authors studied the behavior of EVs of such potentials, focusing primarily on the case where $p(t)=C t^{2}$, the constant $C$ being called the chirp parameter [3]. Generically (but not always) the EVs are purely imaginary, even though $q(t)$ is complex, and move with increasing $C$ towards absorption into the lower-half plane at $\xi=0$. It can happen, however, that imaginary EVs collide, form a double imaginary EV, split, and move off the imaginary axis. It turns out that the flatness of $q_{0}(t)$ has a bearing on whether EV collisions can occur. There are some known [15] WKB bounds on the width of a strip about the imaginary axis which contains the EVs of (1) in the semiclassical case, where the potential is large. The single-lobe theorem places the EVs exactly on the imaginary axis.

\section{APPENDIX}

We show that a double EV occurs exactly when Eq. (16) holds. Let $\xi$ be an EV with eigenfunction

$$
\vec{\nu}(t, \xi)=\left(\begin{array}{l}
\nu_{1}(t, \xi) \\
\nu_{2}(t, \xi)
\end{array}\right)
$$

Since $\vec{\nu}(t, \xi)$ is a multiple of each of $\vec{\psi}(t, \xi)$ and $\vec{\varphi}(t, \xi)$ then Eq. (16) is equivalent to the same equation with $\vec{\nu}(t, \xi)$ replaced by $\vec{\psi}(\xi, t)$. In the Wronskian $W(\xi)=W[\vec{\psi}, \vec{\varphi}](t, \xi)$, which is independent of $t$ by Eq. (1), we have $\vec{\varphi}(t, \xi)$ $=C \vec{\psi}(t, \xi)$ for some constant $C \neq 0$. Differentiating $W(\xi)$ with respect to $\xi$, using a dot to denote the $\xi$ derivative, 


$$
\dot{W}(\xi)=W[\dot{\vec{\psi}}, \vec{\varphi}]+W[\vec{\psi}, \dot{\vec{\varphi}}]=C W[\dot{\vec{\psi}}, \vec{\psi}]+(1 / C) W[\vec{\varphi}, \dot{\vec{\varphi}}]
$$

an expression that is independent of $t$ (even if $\xi$ were not an EV). By Eq. (1), $\left(\dot{\psi}_{1} \psi_{2}-\dot{\psi}_{2} \psi_{1}\right)^{\prime}=-2 i \psi_{1} \psi_{2}$ and so

$$
\begin{aligned}
& W[\dot{\vec{\psi}}, \vec{\psi}](X, \xi)-W[\dot{\vec{\psi}}, \vec{\psi}](-X, \xi) \\
& \quad=\dot{\psi}_{1} \psi_{2}-\left.\dot{\psi}_{2} \psi_{1}\right|_{-X} ^{X} \\
& \quad=-2 i \int_{-X}^{X} \psi_{1} \psi_{2} d t
\end{aligned}
$$

where the $X$ and $-X$ in Eq. (A2) refer to $t$ values. By Eq. (3) the functions $\vec{\psi}$ and $\vec{\varphi}$ are exponentially decreasing in the respective directions, and the same can be established for their $\xi$ derivatives. Therefore,

$$
W[\dot{\vec{\psi}}, \vec{\psi}](X, \xi) \rightarrow 0, \quad W[\vec{\varphi}, \dot{\vec{\varphi}}](-X, \xi) \rightarrow 0, \quad X \rightarrow \infty
$$

so the first term in Eq. (A2) tends to 0. Since Eq. (A1) is independent of $t$,

$$
\dot{W}(\xi)=\lim _{X \rightarrow \infty} C W[\dot{\vec{\psi}}, \vec{\psi}](-X, \xi)+\lim _{X \rightarrow \infty}(1 / C) W[\vec{\varphi}, \dot{\vec{\varphi}}](-X, \xi)
$$

the second limit of which is 0 by Eq. (A3). Using Eqs. (A1)(A4),

$$
\dot{W}(\xi)=\lim _{X \rightarrow \infty} C W[\dot{\vec{\psi}}, \vec{\psi}](-X, \xi)=2 i C \int_{-\infty}^{\infty} \psi_{1} \psi_{2} d t
$$

Therefore Eq. (16) is equivalent to $\dot{W}(\xi)=0$, that is, a double EV.
[1] M. Ablowitz and H. Segur, Solitons and the Inverse Scattering Transform (SIAM, Philadelphia, 1981).

[2] S. Novikov, S. V. Manikov, L. P. Pitaevskii, and V. E. Zakharov, Theory of Solitons: The Inverse Scattering Method (Consultants Bureau, New York, 1984).

[3] G. P. Agrawal, Nonlinear Fiber Optics, 2nd ed. (Academic, New York, 1995).

[4] Z. V. Lewis, Phys. Lett. 112A, 99 (1985).

[5] K. J. Blow and D. Wood, Opt. Commun. 58, 349 (1986).

[6] C. Desem and P. L. Chu, Opt. Lett. 11, 248 (1986).

[7] J. Satsuma and N. Yajima, Suppl. Prog. Theor. Phys. 55, 284 (1974).

[8] D. Anderson, M. Lisak, and T. Reichel, J. Opt. Soc. Am. B 5,
207 (1988).

[9] A. I. Maimistov and Y. M. Sklyarov, Kvant. Elektron. 14, 796 (1987) [Sov. J. Quantum Electron. 17, 500 (1987)].

[10] M. N. Belov, Kvant. Elektron. 14, 1627 (1987) [Sov. J. Quantum Electron. 17, 1033 (1987)].

[11] J. W. Miles, SIAM (Soc. Ind. Appl. Math.) J. Appl. Math. 41, 227 (1981).

[12] M. Klaus and J. K. Shaw, Opt. Commun. 197, 491 (2001).

[13] M. Jaworski, Phys. Rev. E 56, 6142 (1997).

[14] V. E. Zakharov and A. B. Shabat, Zh. Eksp. Teor. Fiz. 61, 118 (1971) [Sov. Phys. JETP 34, 62 (1972)].

[15] J. C. Bronski, Physica D 97, 376 (1996). 